\title{
Recursive fast computation of the two-dimensional discrete cosine transform
}

\author{
W.-H.Fang \\ N.-C.Hu \\ S.-K.Shih
}

\begin{abstract}
An efficient algorithm is presented for computing the two-dimensional discrete cosine transform (2-D DCT) whose size is a power of a prime. Based on a generalised 2-D to onedimensional (1-D) index mapping scheme, the proposed algorithm decomposes the 2-D DCT outputs into three parts. The first part forms a 2 D DCT of a smaller size. The remaining outputs are further decomposed into two parts, depending on the summation of their indices. The latter two parts can be reformulated as a set of circular correlation (CC) or skew-circular correlation (SCC) matrix-vector products by utilising the recently addressed maximum coset decomposition. Such a decomposition procedure can be repetitively carried out for the 2-D DCT of the first part, resulting in a sequence of $\mathrm{CC}$ and $\mathrm{SCC}$ matrix-vector products of various sizes. Employing fast algorithms for the computation of these CC/SCC operations can substantially reduce the numbers of multiplications compared with those of the conventional row-column decomposition approach. In the special case where the data size is a power of two, the proposed algorithm can be further simplified, calling for computations comparable with those of previous works.
\end{abstract}

\section{Introduction}

The DCT has found applications in various facets of signal-processing applications, ranging from transform domain adaptive filtering and the design of filter banks to speech coding [1]. The 2-D DCT, in particular, has been widely considered to be the most effective scheme for transform coding. As such, various international standards, such as the MPEG-1,2 and H.261, have incorporated the 2-D DCT as the image/video coding ingredient block. To facilitate real-time implementations, the development of efficient algorithms for computing the 2-D DCT has been of great interest over the past few decades.

The conventional approach for computing the 2-D DCT is based on the row-column decomposition

(C) IEE, 1999

IEE Proceedings online no. 19990021

DOI: $10.1049 /$ ip-vis: 19990021

Paper first received 5th May and in revised form 26th October 1998

The authors are with the Department of Electronic Engineering, National Taiwan University of Science \& Technology, Taipei, Taiwan, Republic of China method, which first determines the row (column) transforms of the input 2-D data, then transposes these intermediate results, and finally takes the column (row) transforms of the transposed data.

More efficient algorithms can, in general, be attained by working directly on the 2-D data by fully exploiting the 2-D characteristic of the kernel function. For example, Cho and Lee addressed a fast algorithm which decomposes an $N \times N$ DCT into $N$ 1-D $N$-point DCTs [2], in contrast to the $2 N \mathrm{~N}$-point DCTs required for the row-column approach. Duhamel and Guillemot considered an efficient polynomial transform-based approach $[3,4]$. Wu and Paoloui proposed a recursive 2-D DCT algorithm [5] that extended Hou's work [6]. Kung and Lee considered a convolution-based algorithm that admits efficient hardware implementations [7]. Despite their efficiency, these fast algorithms, however, are only applicable to the 2-D DCT of size $2^{r} \times$ $2^{r}$.

In this paper, we address a new fast algorithm for the recursive computation of the 2-D DCT of size $p^{r} \times p^{r}$, where $p$ is a prime. The essence of the new algorithm is based on the 2-D to 1-D index mapping scheme, as addressed in [8]. This index mapping scheme has successfully been applied to the fast computation of the 2D generalised discrete Hartley transform. A similar technique was also utilised to compute the 2-D discrete Fourier transform using the discrete Randon transform (or linear congruence) [9]. Based on this index mapping scheme, the proposed algorithm decomposes the 2-D DCT output components into three parts. In the first part, the output components whose indices are multiples of $p$ form a $p^{r-1} \times p^{r-1}$ DCT. The remaining output components are further decomposed into two parts, depending on the summation of their indices. The latter two parts can be reformulated as a set of CC or SCC matrix-vector products by utilising the recently proposed maximum coset decomposition $[10,11]$. Such a decomposition procedure can be repetitively carried out for the 2-D DCT of the first part by following the same steps. As a consequence, the computation of the 2-D DCT is converted into a sequence of CC and SCC matrix-vector products of various sizes. Employing fast algorithms for the computation of these $\mathrm{CC} / \mathrm{SCC}$ operations, we can thus substantially reduce the numbers of multiplications required, compared with those of the row-column decomposition approach. In the special case when $p=2$, the proposed algorithm can be further simplified, calling for computations comparable with those of previous works.

\section{Fast algorithms for computing the 2-D DCT}

For a set of $2-\mathrm{D}$ data $\left\{x\left(k_{1}, k_{2}\right) ; 0 \leq k_{1} \leq N-1,0 \leq k_{2}\right.$ $\leq N-1\}$, the 2-D DCT is defined as 


$$
\begin{gathered}
X\left(n_{1}, n_{2}\right)=\sum_{k_{1}=0}^{N-1} \sum_{k_{2}=0}^{N-1} x\left(k_{1}, k_{2}\right) \cos \left(\frac{2 \pi\left(2 k_{1}+1\right) n_{1}}{4 N}\right) \\
\times \cos \left(\frac{2 \pi\left(2 k_{2}+1\right) n_{2}}{4 N}\right)
\end{gathered}
$$

for $0 \leq n_{1}, n_{2}, \leq N-1$, where the scale factor, without loss of generality, has been left out for notational brevity, and $N=p^{r}$ with $p$ being a prime. Invoking the trigonometric identity of $\cos (\alpha) \cos (\beta)=1 / 2[\cos (\alpha+\beta)+$ $\cos (\alpha-\beta)$ ], we can rewrite eqn. 1 as

$$
X\left(n_{1}, n_{2}\right)=\frac{1}{2}\left[X^{+}\left(n_{1}, n_{2}\right)+X^{-}\left(n_{1}, n_{2}\right)\right]
$$

where

$$
\begin{aligned}
X^{+}\left(n_{1}, n_{2}\right) & =\sum_{k_{1}=0}^{N-1} \sum_{k_{2}=0}^{N-1} x\left(k_{1}, k_{2}\right) \\
& \times \cos \left(\frac{2 \pi\left[\left(2 k_{1}+1\right) n_{1}+\left(2 k_{2}+1\right) n_{2}\right]}{4 N}\right)
\end{aligned}
$$

and

$$
\begin{aligned}
X^{-}\left(n_{1}, n_{2}\right) & =\sum_{k_{1}=0}^{N-1} \sum_{k_{2}=0}^{N-1} x\left(k_{1}, k_{2}\right) \\
& \times \cos \left(\frac{2 \pi\left[\left(2 k_{1}+1\right) n_{1}-\left(2 k_{2}+1\right) n_{2}\right]}{4 N}\right)
\end{aligned}
$$
[8],

Using the 2-D to 1-D index mapping equations of

$$
\begin{array}{rlr}
\left(2 k_{1}+1\right) n_{1} \pm\left(2 k_{2}+1\right) n_{2} \equiv(2 n+1) 2 k \bmod & 4 N \\
& \text { if } n_{1}+n_{2} \text { is even } & (5) \\
\left(2 k_{1}+1\right) n_{1} \pm\left(2 k_{2}+1\right) n_{2} \equiv(2 n+1)(2 k+1) \bmod & 4 N \\
\text { otherwise } & (6)
\end{array}
$$

$X^{+}\left(n_{1}, n_{2}\right)$ and $X^{-}\left(n_{1}, n_{2}\right)$ can then be evaluated, respectively, using the following 1-D expressions:

$$
\begin{aligned}
X^{+}\left(n_{1}, n_{2}\right) & \triangleq Y^{+}(n) \\
= & \left\{\begin{array}{c}
\sum_{k=0}^{\frac{N+1}{2}-1} y^{+}(k) \cos \left(\frac{2 \pi(2 n+1) 2 k}{4 N}\right) \\
\text { if } n_{1}+n_{2} \text { is even } \\
\sum_{k=0}^{\frac{N-1}{2}-1} y^{+}(k) \cos \left(\frac{2 \pi(2 n+1)(2 k+1)}{4 N}\right) \\
\text { otherwise }
\end{array}\right.
\end{aligned}
$$

and

$$
\begin{aligned}
X^{-}\left(n_{1}, n_{2}\right) & \triangleq Y^{-}(n) \\
= & \left\{\begin{array}{l}
\sum_{k=0}^{\frac{N+1}{2}-1} y^{-}(k) \cos \left(\frac{2 \pi(2 n+1) 2 k}{4 N}\right) \\
\text { if } n_{1}+n_{2} \text { is even } \\
\sum_{k=0}^{\frac{N-1}{2}-1} y^{-}(k) \cos \left(\frac{2 \pi(2 n+1)(2 k+1)}{4 N}\right) \\
\text { otherwise }
\end{array}\right.
\end{aligned}
$$

where $y^{ \pm}(k)=\Sigma_{k_{1}} \Sigma_{k_{2}} x\left(k_{1}, k_{2}\right)$, in which $\left(k_{1}, k_{2}\right)$ satisfies the index mapping equations of eqn. 5 or eqn. 6 , depending on whether $n_{1}+n_{2}$ is even or not. Thereby, $X\left(n_{1}, n_{2}\right)$ can now be rewritten as

$$
\begin{aligned}
& X\left(n_{1}, n_{2}\right) \triangleq Y(n) \\
& =\left\{\begin{array}{cc}
\sum_{k=0}^{\frac{N+1}{2}-1} y(k) \cos \left(\frac{2 \pi(2 n+1) 2 k}{4 N}\right) \\
\text { if } n_{1}+n_{2} \text { is even } \\
\sum_{k=0}^{\frac{N-1}{2}-1} y(k) \cos \left(\frac{2 \pi(2 n+1)(2 k+1)}{4 N}\right) \\
\text { otherwise }
\end{array}\right.
\end{aligned}
$$

where $Y(n) \triangleq 1 / 2\left[Y^{+}(n)+Y(n)\right]$ and $y(k) \triangleq 1 / 2\left[y^{+}(k)+\right.$ $\left.y^{-}(k)\right]$. Next, we address the issue of solving the 2-D to 1-D index mapping equations (eqns. 5 and 6). To solve these, $n_{1}, n_{2}$ and $n$ must be decided first. In other words, the index $\left(n_{1}, n_{2}\right)$ of the 2-D output component is first mapped to a predetermined 1-D output component with an index $n$, denoted by $\left(n_{1}, n_{2}\right) \rightarrow n$. According to this $\left(n_{1}, n_{2}\right) \rightarrow n$, a solution set of $\left(k_{1}, k_{2}\right)$ on the left-hand side of eqns. 5 and 6 can thus be obtained for every specific $k$ on the right-hand sides of eqns. 5 and 6. $y(k)$ can then be determined by a summation of $x\left(k_{1}\right.$, $k_{2}$ ) of these corresponding $\left(k_{1}, k_{2}\right) \mathrm{s}$.

To mitigate the overhead in the computation of $y(k)$, we consider those $\left\{X\left(n_{1}, n_{2}\right)\right\}$ that share the same set of $\{y(k)\}$ together. As all of the corresponding $\left(n_{1}, n_{2}\right) \mathrm{s}$ satisfy the congruence eqns. 5 and 6 , they are related to each other by some factors. Therefore, if one of these indices, say $\left(n_{1}^{0}, n_{2}^{0}\right), \rightarrow 0$, then the other indices $\left(n_{1}\right.$, $n_{2}$ ) s inside this set can be determined as a function of $\left(n_{1}^{0}, n_{2}^{0}\right)$ and $n$. Such a particular $\left(n_{1}^{0}, n_{2}^{0}\right)$ is thus referred to as a dominant point for the corresponding set of $\left\{X\left(n_{1}, n_{2}\right)\right\}$. Then, as shown in the Appendix, it follows that the rest of $\left\{X\left(n_{1}, n_{2}\right)\right\}$, which share the same set of $\{y(k)\}$, are related to $Y(n), n=1,2, \ldots$, $(N-1) / 2-1$, by

$$
Y(n)=X\left(\left\langle(2 n+1) n_{1}^{0}\right\rangle,\left\langle(2 n+1) n_{2}^{0}\right\rangle\right)
$$

where $\langle\cdot\rangle$ stands for either $\bmod 4 N$ or $\bmod 2 N$, depending on whether eqn. 5 or eqn. 6 is employed. Note that, as both $4 N$ and $2 N$ are even for all $N$, it is easy to justify that, if a dominant point $\left(n_{1}^{0}, n_{2}^{0}\right)$ has a summation of indices $n_{1}^{0}+n_{2}^{0}$ that is even(odd), then the corresponding $\left\{X\left(n_{1}, n_{2}\right)\right\}$ has summations of indices $n_{1}+n_{2}$ that are all even(odd).

Some output components, however, appear repetitively for different dominant points, if $(2 n+1)$ is a multiple of $p$. For example, if $N=9$ and for the dominant point $(1,1) \rightarrow 0$, we obtain a corresponding output component set as $Y(0)=X(1,1), Y(1)=X(3,3)$, $Y(2)=X(5,5)$ and $Y(3)=X(7,7)$. On the other hand, the dominant point $(1,5) \rightarrow 0$ will generate another output component set as $Y(0)=X(1,5), Y(1)=X(3,3)$, $Y(2)=X(5,7)$, and $Y(3)=X(7,1)$. We can note that $X(3,3)$ is repetitively computed, thus increasing the computational load. To avoid this redundant computation, $n$ that satisfies $(2 n+1)=m p$, or equivalently, when both indices of the outputs are multiples of $p$ owing to eqn. 10, will be treated separately. Given this fact, along with the index mapping eqns. 5 and 6 , we can classify the computation of the 2-D DCT in the following three categories, according to the output indices:

Part 1: $\left\{X\left(n_{1}, n_{2}\right): n_{1} \bmod p=n_{2} \bmod p=0\right\}$

Part 2: $\left\{X\left(n_{1}, n_{2}\right): n_{1} \bmod p \neq 0\right.$ or $n_{2} \bmod p \neq 0, n_{1}+$ $n_{2}$ is even

IEE Proc.-Vis. Image Signal Process. Vol. 146. No. 1. February 1999 
Part 3: $\left\{X\left(n_{1}, n_{2}\right): n_{1} \bmod p \neq 0\right.$ or $n_{2} \bmod p \neq 0, n_{1}+$ $n_{2}$ is odd $\}$

For the output components of part 2, using the maximum coset decomposition as addressed in $[10,11]$, the corresponding $2 n+1(2 n+1 \neq m p)$ can be obtained by a generator that is an odd prime. More specifically, such a set of numbers can be generated by a power of $g$ $\bmod 2 N$ as $G_{1}=\left\{g^{0}, g^{1}, \ldots, g^{\lambda-1}\right\} \bmod 2 N$, where $g$ is the coset generator with order $\lambda$ that satisfies $\cos (2 \pi /$ $4 N)= \pm \cos \left(2 \pi g^{r} / 4 N\right)$ Therefore, for every dominant point, there are only $\lambda$ (non-redundant) corresponding $Y(n)$ s (or, equivalently, $\left.X\left(n_{1}, n_{2}\right) \mathrm{s}\right)$, where $\lambda=p^{r-1}(p-$ 1) $/ 2$, if $N=p^{r}$ [12]. Also, the $(N+1) / 2$ input points in eqn. $9 a$ can be divided into the following cosets: $G_{1}, G_{2}$ $=p G_{1}=\left\{p, p g, \ldots, p g^{\lambda / p-1}\right\} \bmod 2 N, \ldots, G_{r}=p^{r-1} G_{1}$ $\bmod 2 N$, and $G_{r+1}=\{N\}$. The corresponding orders of these cosets are $\lambda, \lambda / p, \ldots$, and 1 , respectively, and their summation is equal to $\lambda+\lambda / p+\ldots+1=(N+1) / 2$.

By replacing $(2 n+1)$ and $k$ with $g^{m}$ and $p^{i} g^{l}$, respectively, in eqn. $9 a$, we can rewrite eqn. $9 a$ with $(N+1)$ 2 inputs and $\lambda$ outputs as

$$
\begin{aligned}
Y\left(\left\langle\frac{g^{m}-1}{2}\right\rangle\right) \\
\quad=\sum_{i=0}^{r} \sum_{l=0}^{\lambda / p^{i}-1} y\left(\left\langle p^{i} g^{l}\right\rangle\right) \cos \left(\frac{2 \pi p^{i} g^{l+m}}{2 N}\right)
\end{aligned}
$$

where $m=0,1, \ldots, \lambda-1$, and $\langle\cdot\rangle$ denotes $\bmod 2 N$ operation. Expanding eqn. 11 yields the following more illustrative matrix notation:

$$
\begin{aligned}
& \left.\left[\begin{array}{c}
Y\left(\left\langle\frac{g^{0}-1}{2}\right\rangle\right) \\
Y\left(\left\langle\frac{g^{1}-1}{2}\right\rangle\right) \\
Y\left(\left\langle\frac{g^{2}-1}{2}\right\rangle\right) \\
\vdots \\
Y\left(\left\langle\frac{g^{\lambda}-1}{2}-1\right.\right. \\
\end{array}\right]\right) \\
& =\sum_{i=0}^{r}\left[\begin{array}{cccc}
C_{p^{i} g^{0}} & C_{p^{i} g^{1}} & \cdots & C_{p^{i} g^{\lambda / p^{i}-1}} \\
C_{p^{i} g^{1}} & C_{p^{i} g^{2}} & \cdots & C_{p^{i} g^{0}} \\
C_{p^{i} g^{2}} & \cdots & \cdots & C_{p^{i} g^{1}} \\
\vdots & \vdots & \cdots & \vdots \\
C_{p^{i} g^{\lambda-1}} & C_{p^{i} g^{0}} & \cdots & C_{p^{i} g^{\lambda-2}}
\end{array}\right] \\
& \times\left[\begin{array}{c}
y\left(\left\langle p^{i} g^{0}\right\rangle\right) \\
y\left(\left\langle p^{i} g^{1}\right\rangle\right) \\
y\left(\left\langle p^{i} g^{2}\right\rangle\right) \\
\vdots \\
y\left(\left\langle p^{i} g^{\lambda-1}\right\rangle\right)
\end{array}\right]
\end{aligned}
$$

where $C_{k} \triangleq \cos (2 \pi k / 2 N)$ and we have used the fact that $C_{p} i_{g}^{\lambda} p^{i}+m=C_{p}{ }^{i} g^{m}, i=0, \ldots, r, m=0, \ldots, \lambda-1$. As such, the computation of the 2-D DCT output components in part 2 can be implemented by a sequence of $\mathrm{CC}$ matrix-vector products of sizes $\lambda / p^{i}, i=0, \ldots, r$.

Note that, as $0 \leq\left\langle g^{m}\right\rangle \bmod 2 N$, some rectifications are made for the $y(k)$ in eqn. $9 a$. If $\left\langle p^{i} g^{m}\right\rangle<(N+1) / 2$ then we simply use $y\left(\left\langle p^{i} g^{m}\right\rangle\right)$; on the other hand, if $\left\langle p^{i} g^{m}\right\rangle \geq(N+1) / 2$ we will use $-y\left(N-\left\langle p^{i} g^{m}\right\rangle\right)$, as readily justified using eqns. 5 and 6 . Similarly, if $\left\langle g^{i}\right\rangle<N$, the output of eqn. $9 b$ is $Y\left(\left\langle\left(g^{i}-1\right) / 2\right\rangle\right)$; otherwise, the output will become $-Y(n)$, where $\left\langle g^{i}\right\rangle=2 N-(2 n+1)$.
Along the same line, the computation of the output components of part 3 can be simplified by following the above steps, except that the operation of $\bmod 2 N$ is replaced with $\bmod 4 N$. More specifically, for every dominant point, the corresponding $2 n+1$ can be generated by a generator $g \bmod 4 N$ as $G_{1}=\left\{g^{0}, g^{1}, \ldots\right.$, $\left.g^{\lambda-1}\right\} \bmod 4 N$, where $g$ is the coset generator with order $\lambda$. The $(N-1) / 2$ input points in eqn. $9 b$ can thus be divided into the following cosets: $G_{1}, G_{2}=p G_{1}=\{p$, $\left.p g, \ldots, p g^{\lambda / p-1}\right\} \bmod 4 N, . .$, and $G_{r}=p^{r-1} G_{1} \bmod 4 N$. The corresponding orders of these cosets are $\lambda, \ldots$, and $\lambda /\left(p^{r-1}\right)$, respectively, and their summation is equal to $\lambda$ $+\lambda p+\ldots+\lambda p^{r-1}=(N-1) / 2$.

By replacing $(2 n+1)$ and $(2 k+1)$ with $g^{m}$ and $p^{i} g^{l}$, respectively, in eqn. $9 b$, we can rewrite eqn. $9 b$ with $(N-1) / 2$ inputs and $\lambda$ outputs as

$$
\begin{aligned}
& Y\left(\left\langle\frac{g^{m}-1}{2}\right\rangle\right) \\
& \quad=\sum_{i=0}^{r-1} \sum_{k=0}^{\frac{\lambda}{p^{i}}-1} y\left(\frac{\left\langle p^{i} g^{k}\right\rangle-1}{2}\right) \cos \left(\frac{2 \pi p^{i} g^{l+m}}{4 N}\right)
\end{aligned}
$$

where $m=0,1, \ldots, \lambda-1$ and $\langle\cdot\rangle$ denotes $\bmod 4 N$ operation. As above, we can also express eqn. 13 as the following matrix notation:

$$
\begin{aligned}
& {\left[\begin{array}{c}
Y\left(\left\langle\frac{g^{0}-1}{2}\right\rangle\right) \\
Y\left(\left\langle\frac{g^{1}-1}{2}\right\rangle\right) \\
Y\left(\left\langle\frac{g^{2}-1}{2}\right\rangle\right) \\
\vdots \\
Y\left(\left\langle\frac{g^{\lambda}-1}{2}\right\rangle\right)
\end{array}\right]} \\
& =\sum_{i=0}^{r-1}\left[\begin{array}{cccc}
\bar{C}_{p^{i} g^{0}} & \bar{C}_{p^{i} g^{1}} & \cdots & \bar{C}_{p^{i} g^{\lambda / p^{i}-1}} \\
\bar{C}_{p^{i} g^{1}} & \bar{C}_{p^{i} g^{2}} & \cdots & -\bar{C}_{p^{i} g^{0}} \\
\bar{C}_{p^{i} g^{2}} & \cdots & \cdots & -\bar{C}_{p^{i} g^{1}} \\
\vdots & \vdots & \vdots & \vdots \\
\bar{C}_{p^{i} g^{\lambda-1}} & -\bar{C}_{p^{i} g^{0}} & \cdots & -\bar{C}_{p^{i} g^{\lambda-2}}
\end{array}\right] \\
& \times\left[\begin{array}{c}
y\left(\frac{\left\langle p^{i} g^{0}\right\rangle-1}{2}\right) \\
y\left(\frac{\left\langle p^{i} g^{1}\right\rangle-1}{2}\right) \\
y\left(\frac{\left\langle p^{i} g^{2}\right\rangle-1}{2}\right) \\
\vdots \\
y\left(\frac{\left\langle p^{i} g^{\dot{\lambda}}-1\right.}{2}\right\rangle-1 \\
2
\end{array}\right]
\end{aligned}
$$

where $\bar{C}_{k} \triangleq \cos ((2 \pi k) / 4 N)$, and we have used the fact that $\bar{C}_{p^{i} g^{\lambda} p^{i+m}}=-\bar{C}_{p^{i} g^{m}}, i=0, \ldots, r, m=0, \ldots, \lambda-1$. As such, the computations of the 2-D DCT outputs in part 3 can be implemented by a sequence of SCC matrixvector products of sizes $\lambda / p^{i}, i=0, \ldots, r$.

Likewise, the input and output indices are required to be relocated if they are outside the range. If $\left\langle\left(\left\langle p^{i} g^{m}\right\rangle\right.\right.$ $-1) / 2\rangle\left\langle(N-1) / 2\right.$, then the input is $y\left(\left(\left\langle p^{i} g^{m}\right\rangle-1\right) / 2\right)$; on the other hand, if $\left\langle p^{i} g^{m}\right\rangle \geq(N-1) / 2$, we will use the input as $-y\left(N-\left(\left\langle p^{i} g^{m}\right\rangle\right) / 2\right)$ in eqn. $9 b$. Also, if $\left\langle\left(g^{i}-\right.\right.$ $1) / 2\rangle<N$, the output of eqn. $9 b$ is $Y\left(\left\langle\left(g^{i}-1\right) / 2\right\rangle\right)$; otherwise, the output will become $-Y(n)$ or $Y(n)$, depending on whether $\left\langle g^{\mathrm{i}}\right\rangle$ can be expressed as $2 N \pm$ $(2 n+1)$ or $4 N-(2 n+1)$. 
From the above discussion, we can note that the algorithm initially requires $\left(N^{2}\left(P^{2}-1\right)\right) / p^{2} \lambda$ dominant points that, in turn, generate a total of $N^{2}\left(1-1 / p^{2}\right)$ output points. Such a decomposition procedure can be recursively carried out for the 2-D DCT of part 1 . The overall steps can be summarised as the following algorithm for computing the 2-D DCT of size $p^{r} \times p^{r}$ (with initial value $i=1$ ):

Step 1: Divide the output components into three parts, according to their indices. If both of the indices $n_{1}$ and $n_{2}$ are multiples of $p$ (part 1), these $\left\{X\left(n_{1}, n_{2}\right)\right\}$ form a 2-D DCT of size $p^{r-i} \times p^{r-i}$. The rest of the output components can be further classified into two parts, part 2 and part 3 , depending on whether the summation of $n_{1} /\left(p^{i-1}\right)+n_{2} /\left(p^{i-1}\right)$ is even or odd, respectively.

Step 2: The $\left\{X\left(n_{1}, n_{2}\right)\right\}$ in parts 2 and 3 can be further classified into several groups with different choices of dominant points. Every group of $\left\{X\left(n_{1}, n_{2}\right)\right\}$ in parts 2 and 3 can be implemented using the $C C$ operation of eqn. 12 and the SCC operation of eqn. 14, respectively. The resulting output components can be obtained by using the index mapping scheme of eqn. 10. The $p^{r-i} \times p^{r-i}$ DCT of part 1 can be further decomposed along the same steps by going back to step 1 , with $i=i+1$ until $i=r$.

As a special case when $p=2$, eqn. $9 a$ and $b$ can be simplified as

$$
X\left(n_{1}, n_{2}\right)=\left\{\begin{array}{cc}
\sum_{k=0}^{\frac{N+1}{2}-1} y(k) \cos \left(\frac{2 \pi(2 n+1) 2 k}{4 N}\right) \\
\text { if } n_{1}+n_{2} \text { is even } \\
\sum_{k=0}^{\frac{N}{2}-1} y(k) \cos \left(\frac{2 \pi(2 n+1)(2 k+1)}{4 N}\right) \\
\text { otherwise }
\end{array}\right.
$$

Employing the maximum coset decomposition as discussed above, we can obtain

$$
\begin{array}{r}
Y\left(\left\langle\frac{g^{m}-1}{2}\right\rangle\right) \\
=\sum_{i=0}^{r} \sum_{l=0}^{\lambda / 2^{i}-1} y\left(\left\langle 2^{i} g^{m}\right\rangle\right) \cos \left(\frac{2 \pi 2^{i} g^{m+l}}{2 N}\right) \\
\text { if } n_{i}+n_{2}=\text { even }
\end{array}
$$

and

$$
Y\left(\left\langle\frac{g^{m}-1}{2}\right\rangle\right)=\sum_{l=0}^{\lambda-1} y\left(\left\langle p^{i} g^{m}\right\rangle\right) \cos \left(\frac{2 \pi p^{i} g^{m+l}}{4 N}\right)
$$

otherwise

where $m=0, \ldots, \lambda-1$, and $\langle\cdot\rangle$ denotes $\bmod 2 N$ and $4 N$ in eqns. 16 and 17 , respectively. As above, eqns. 16 and 17 can be implemented using a sequence of SCC matrix-vector products. These SCC operations can be efficiently implemented via the algorithm of [10], with $y(k)$ as the input and the odd-indexed output components as the corresponding output.

To follow, we consider two examples to reinforce the proposed algorithm.

Example $1(5 \times 5$ DCT $)$ : As $N$ is equal to $5(p=5$ and $r=1)$ in this example, there are $N^{2}\left(1-1 / p^{2}\right)=24$ output components to be determined in the first iteration. If we choose the generator $g=3$, the corresponding order $\lambda$ can be easily found to be 2 . This implies that a total of 12 dominant points, which can be (arbitrarily) chosen as $(0,1),(1,0),(1,2),(1,4),(4,1),(4$, $3),(0,2),(1,1),(1,3),(2,0),(2,2)$ and $(2,4)$, are required. Then, we determine the corresponding $\{y(k)\}$ for the above dominant points by using the index mapping schemes of eqns. 5 and 6.

For the dominant points $(0,2),(1,1),(1,3),(2,0)$, $(2,2)$ and $(2,4)$, as the index summations are even, eqn. 5 is employed. As an example, for the dominant point $(1,1), y(k)=1 / 2\left[y^{+}(k)+y^{-}(k)\right], k=0,1,2$, where $y^{+}(k)$ and $y(k)$ can be determined using eqn. 5 as follows:

$$
\begin{aligned}
y^{+}(0)= & -x(0,4)-x(1,3)-x(2,2)-x(3,1) \\
& -x(4,0) \\
y^{-}(0)= & +x(0,0)+x(1,1)+x(2,2)+x(3,3) \\
& +x(4,4) \\
y^{+}(1)= & +x(0,0)-x(0,3)-x(1,2)-x(1,4) \\
& -x(2,1)-x(2,3)-x(3,0)-x(3,2) \\
& -x(4,1)+x(4,4) \\
y^{-}(1)= & +x(0,1)-x(0,4)+x(1,0)+x(1,2) \\
& +x(2,1)+x(2,3)+x(3,2)+x(3,4) \\
& -x(4,0)+x(4,3) \\
& +x(0,1)-x(0,2)+x(1,0)-x(1,1) \\
& -x(2,0)-x(2,4)-x(3,3)+x(3,4) \\
& -x(4,2)+x(4,3) \\
y^{+}(2)= & +x(0,2)-x(0,3)+x(1,3)-x(1,4) \\
& +x(2,0)+x(2,4)-x(3,0)+x(3,1) \\
& -x(4,1)+x(4,2)
\end{aligned}
$$

An illuminative picture to demonstrate the above computation is shown in Fig. 1. For every one of these dominant points, the corresponding $\{Y(n)\}$, which shares the same $\{y(k)\}$, can be determined using the $\mathrm{CC}$ matrix-vector product of eqn. 12, as follows:

$$
\begin{aligned}
{\left[\begin{array}{l}
Y(0) \\
Y(1)
\end{array}\right]=} & {\left[\begin{array}{ll}
\cos \frac{2 \pi 1}{10} & \cos \frac{2 \pi 3}{10} \\
\cos \frac{2 \pi 3}{10} & \cos \frac{2 \pi 1}{10}
\end{array}\right]\left[\begin{array}{c}
y(1) \\
-y(2)
\end{array}\right] } \\
& +\left[\cos \frac{2 \pi 5}{10}\right][-y(0)]
\end{aligned}
$$

The resulting $\left\{X\left(n_{1}, n_{2}\right)\right\}$ can then be determined using eqn. 10. Following the above for the dominant

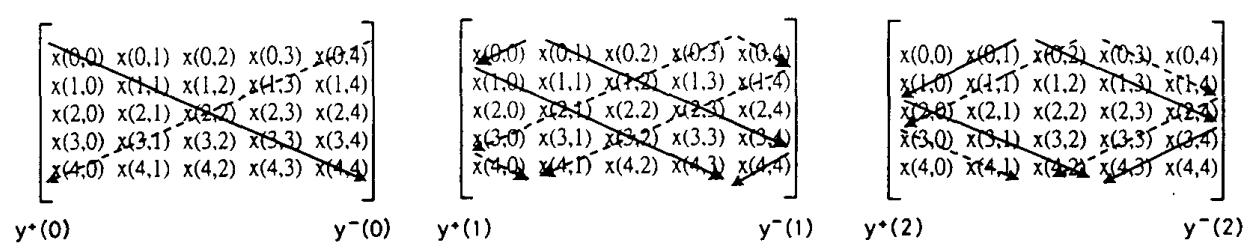

Fig. 1 Computation of $y^{+}(k)$ and $y^{-}(k)$ for dominant point $(1,1)$ 
point $(1,1)$, we obtain

$$
\begin{aligned}
& Y(0)=X\left(n_{1}^{0}, n_{2}^{0}\right)=X(1,1) \\
& Y(1)=X\left(\left\langle 3 n_{1}^{0}\right\rangle,\left\langle 3 n_{2}^{0}\right\rangle\right)=X(3,3)
\end{aligned}
$$

Similarly, for the dominant points $(0,1),(1,0),(1$, $2),(1,4),(4,1)$ and $(4,3)$, eqn. 6 should be employed to determine $\{y(k)\}$. For every one of these dominant points, the corresponding $\{Y(n)\}$, which shares the same $\{y(k)\}$, can be determined using the SCC matrixvector product of eqn. 14 , as follows:

$$
\left[\begin{array}{l}
Y(0) \\
Y(1)
\end{array}\right]=\left[\begin{array}{cc}
\cos \frac{2 \pi 1}{20} & \cos \frac{2 \pi 3}{20} \\
\cos \frac{2 \pi 3}{20} & -\cos \frac{2 \pi 1}{20}
\end{array}\right]\left[\begin{array}{l}
y(0) \\
y(1)
\end{array}\right]
$$

The resulting outputs $\left\{X\left(n_{1}, n_{2}\right)\right\}$ can then be obtained using the index mapping eqn. 10. In the second iteration, there is only one output component, i.e. $X(0,0)$, that needs to be determined, which can be easily determined by $X(0,0)=\sum_{k_{1}=0}^{N-1} \sum_{k_{2}=0}^{\hat{N}_{-1}} x\left(k_{1}, k_{2}\right)$. A butterfly-like structure depicting the above computing procedure is shown in Fig. 2.

Example $2(4 \times 4$ DCT): As $N$ is equal to $4(p=2$ and $r=2)$ in this example, there are $N^{2}\left(1-1 /\left(p^{2}\right)\right)=12$ output components to be determined in the first iteration. If we also choose the generator $g=3$, the corresponding order $\lambda$ can be easily found to be 2 . This implies that a total of six dominant points, which can be (arbitrarily) chosen as $(0,1),(1,0),(1,2),(2,1),(1$, 1) and $(1,3)$, are required.

For the dominant points $(1,1)$ and $(1,3)$, the index summations of the associated $\left\{X\left(n_{1}, n_{2}\right)\right\}$ are even, and the corresponding $\{Y(n)\}$ can be determined using the

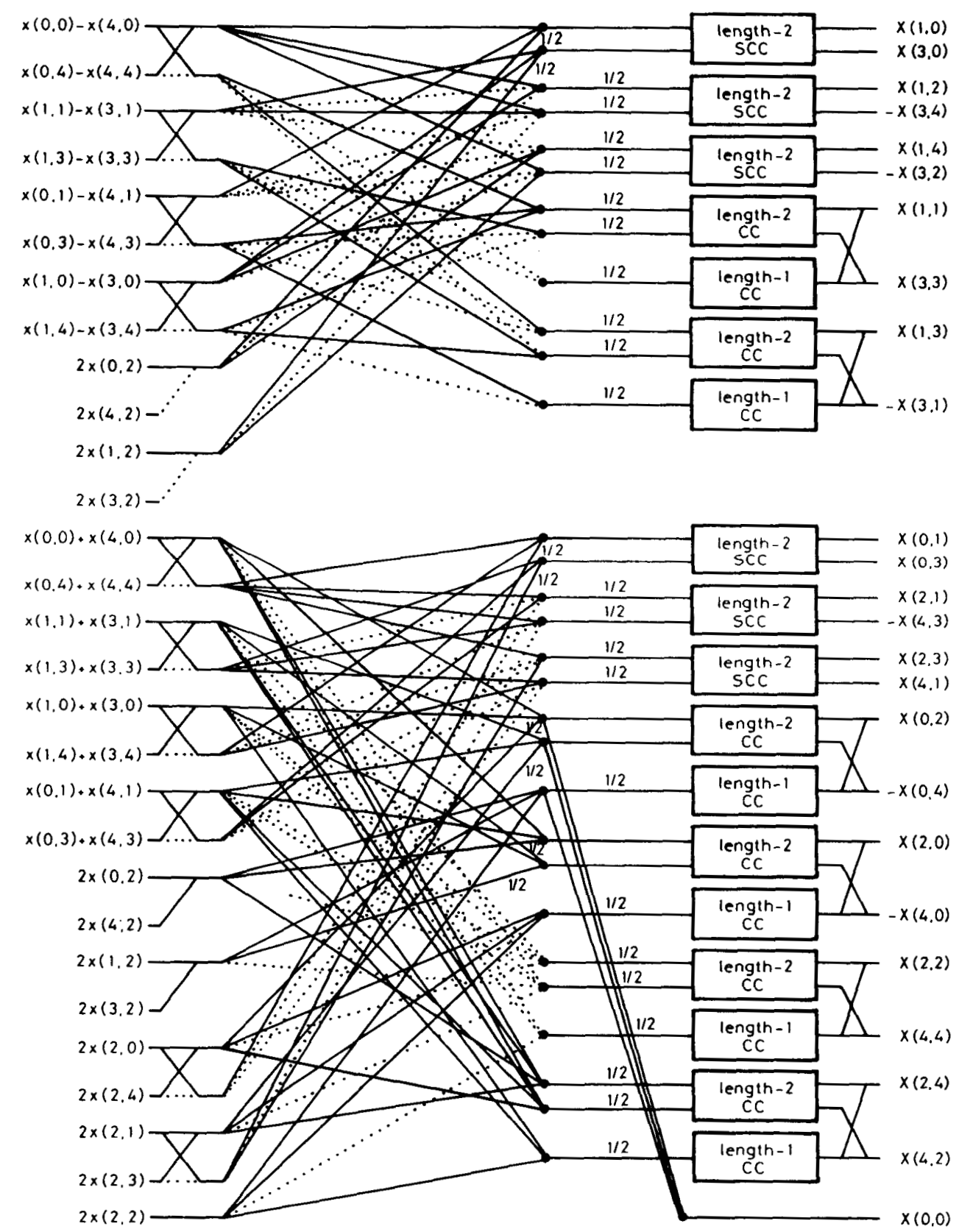

Fig. 2 Structure for computing $5 \times 5$ DCT by using proposed fast algorithm 
CC matrix-vector product of eqn. 12, as follows:

$$
\left[\begin{array}{l}
Y(0) \\
Y(1)
\end{array}\right]=\left[\begin{array}{c}
\cos \frac{2 \pi 1}{8} \\
-\cos \frac{2 \pi 1}{8}
\end{array}\right][y(1)]+y(0)
$$

whereas, for the dominant points $(0,1),(1,0),(1,2)$ and $(2,1)$, the index summations of the associated $\left\{X\left(n_{1}, n_{2}\right)\right\}$ are odd and the corresponding $\{Y(n)\}$ can be determined using the SCC matrix-vector product of eqn. 14 , as follows

$$
\left[\begin{array}{l}
Y(0) \\
Y(1)
\end{array}\right]=\left[\begin{array}{cc}
\cos \frac{2 \pi 1}{16} & \cos \frac{2 \pi 3}{16} \\
\cos \frac{2 \pi 3}{16} & -\cos \frac{2 \pi 1}{16}
\end{array}\right]\left[\begin{array}{l}
y(0) \\
y(1)
\end{array}\right]
$$

In the second iteration, three output components, namely $X(0,2), X(2,0)$ and $X(2,2)$, can be determined by

$$
[Y(0)]=y(1)
$$

and

$$
[Y(0)]=\left[\cos \frac{2 \pi 1}{8}\right][y(0)]
$$

In the third iteration, $X(0,0)$ can be easily obtained by the summation of all inputs $\left\{x\left(k_{1}, k_{2}\right)\right\}$. A butterflylike structure depicting the above computing procedure is shown in Fig. 3.

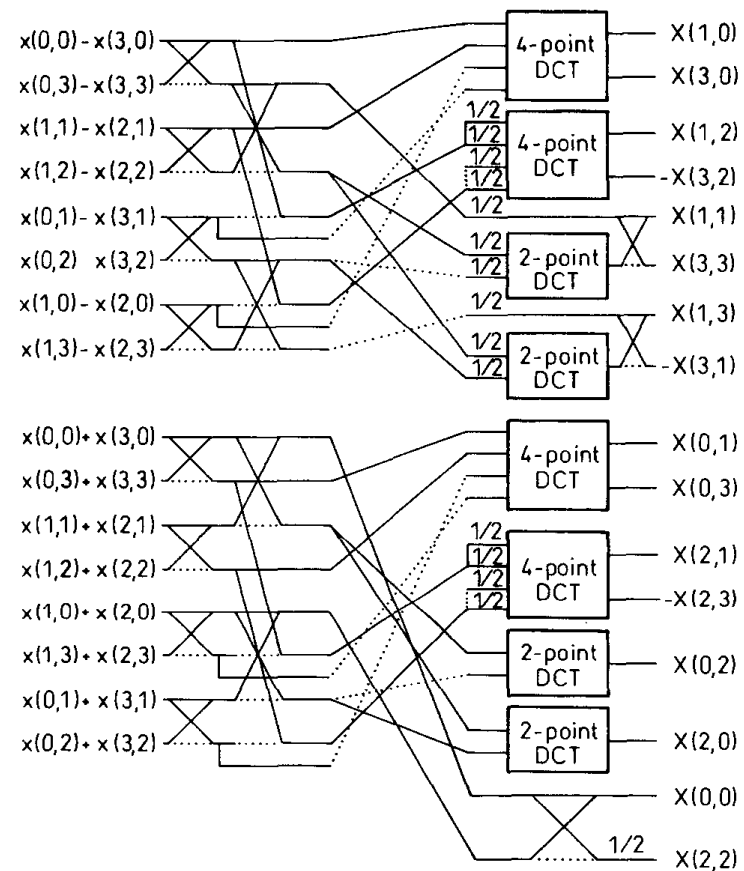

Fig.3 Structure for computing the $4 \times 4 D C T$ by using proposed fast algorithm

For the computation of the 2-D inverse DCT (IDCT), the fast algorithm addressed above can also be employed, with slight modifications. The 2-D IDCT for the transformed data $\left\{X\left(n_{1}, n_{2}\right)\right\}$ is given by

$$
\begin{array}{r}
x\left(k_{1}, k_{2}\right)=\sum_{n_{1}=0}^{N-1} \sum_{n_{2}=0}^{N-1} X\left(n_{1}, n_{2}\right) \cos \left(\frac{2 \pi\left(2 k_{1}+1\right) n_{1}}{4 N}\right) \\
\times \cos \left(\frac{2 \pi\left(2 k_{2}+1\right) n_{2}}{4 N}\right)
\end{array}
$$

for $0 \leq k_{1}, k_{2}, \leq N-1$, where the scale factor has again been left out for brevity, and $N=p^{r}$ with $p$ being a 30 prime. Using the same trigonometric identity, eqn. 24 can be rewritten as

$$
\begin{aligned}
x\left(k_{1}, k_{2}\right)= & \frac{1}{2} \sum_{n_{1}=0}^{N-1} \sum_{n_{2}=0}^{N-1} X\left(k_{1}, k_{2}\right) \\
& \times \cos \left(\frac{2 \pi\left[\left(2 k_{1}+1\right) n_{1}+\left(2 k_{2}+1\right) n_{2}\right]}{4 N}\right) \\
& +\frac{1}{2} \sum_{n_{1}=0}^{N-1} \sum_{n_{2}=0}^{N-1} X\left(k_{1}, k_{2}\right) \\
& \times \cos \left(\frac{2 \pi\left[\left(2 k_{1}+1\right) n_{1}-\left(2 k_{2}+1\right) n_{2}\right]}{4 N}\right)
\end{aligned}
$$

Using a modified 2-D to 1-D index mapping equation, as follows:

$$
\left(2 k_{1}+1\right) n_{1} \pm\left(2 k_{2}+1\right) n_{2} \equiv(2 k+1) 2 n \bmod 4 N
$$

we can re-express eqn. 25 as

$$
\begin{aligned}
x\left(k_{1}, k_{2}\right)= & y(k) \\
= & \sum_{n=0}^{N-1} Y_{1}(n) \cos \left(\frac{2 \pi\left(2 k_{1}+1\right) n}{4 N}\right) \\
& +\sum_{n=0}^{N-1} Y_{2}(n) \cos \left(\frac{2 \pi\left(2 k_{1}+1\right) n}{4 N}\right)
\end{aligned}
$$

where $Y_{1(2)}=\Sigma_{n_{1}} \Sigma_{n_{2}} X\left(n_{1}, n_{2}\right)$ in which $\left(n_{1}, n_{2}\right)$ s satisfy the index mapping equation of eqn. 26 with plus(minus) sign. Employing the maximum coset decomposition scheme addressed above, eqn. 27 can be rewritten as

$$
\begin{aligned}
& y\left(\left\langle\frac{g^{m}-1}{2}\right\rangle\right) \\
& =\sum_{i=0}^{r-1} \sum_{l=0}^{\lambda-1} Y_{1}\left(\left\langle p^{i} g^{m}\right\rangle\right) \cos \left(\frac{2 \pi p^{i} g^{m+l}}{4 N}\right) \\
& \quad+\sum_{i=0}^{r} \sum_{l=0}^{\lambda-1} Y_{2}\left(\left\langle 2 p^{i} g^{m}\right\rangle\right) \cos \left(\frac{2 \pi p^{i} g^{m+l}}{2 N}\right)
\end{aligned}
$$

Expanding eqn. 28 into a matrix form, we can note that the computation of $\{y(k)\}$ is equal to a summation of CC and SCC matrix-vector products, such as those of eqns. 12 and 14 , respectively. In a similar manner, the resulting $\left\{x\left(k_{1}, k_{2}\right)\right\}$ can then be obtained from $\{y(k)\}$ using the index mapping, as follows:

$$
\begin{aligned}
y(k)=x\left(\left\langle\frac{\left\langle(2 k+1)\left(2 k_{1}^{0}+1\right)\right\rangle-1}{2}\right\rangle\right. & \\
& \left.\left\langle\frac{\left\langle(2 k+1)\left(2 k_{2}^{0}+1\right)\right\rangle-1}{2}\right\rangle\right)
\end{aligned}
$$

where $\left(k_{1}^{0}, k_{2}^{0}\right)$ is the chosen dominant point, and $\langle\cdot\rangle$ denotes $\bmod 2 N$ operation.

\section{Comparison and discussion}

In this Section, we compare the computational complexity of the proposed fast algorithm with that of existing ones. Because the algorithm recursively

IEE Proc.-Vis. Image Signal Process. Vol. 146. No. 1, February 1999 
computes the 2-D DCT of size $N \times N\left(N=p^{r}\right)$, it is straightforward to justify that the numbers of multiplications $\operatorname{MUL}(N)$ and additions $\operatorname{ADD}(N)$ are

$$
\begin{aligned}
\operatorname{MUL}(N) & =\sum_{i=0}^{r-1} \frac{N^{2}\left(1-\frac{1}{p^{2}}\right)}{2 \lambda p^{i}} \\
& \times \sum_{j=0}^{r-i-1}\left[M_{c c}\left(\frac{\lambda}{p^{r-j-1}}\right)+M_{s c c}\left(\frac{\lambda}{p^{r-j-1}}\right)\right]
\end{aligned}
$$

and

$$
\begin{aligned}
& \operatorname{ADD}(N)=\sum_{i=0}^{r-1} \frac{N^{2}\left(1-\frac{1}{p^{2}}\right)}{2 \lambda p^{i}} \\
& \times\left\{\sum _ { j = 0 } ^ { r - i - 1 } \left[A_{C C}\left(\frac{\lambda}{p^{r-j-1}}\right)+A_{S C C}\left(\frac{\lambda}{p^{r-j-1}}\right)\right.\right. \\
& \left.\left.+\frac{2 \lambda\left(1+\frac{1}{p}-\frac{1}{p^{2}}\right)}{p^{r-j-1}}\right]-\frac{2 \lambda\left(\frac{1}{2}+\frac{1}{p}-\frac{1}{p^{2}}\right)}{p^{r-1}}\right\} \\
& +\left(\frac{\lambda}{\frac{N}{p}}-1\right)+N^{3}\left(1+\frac{1}{p}\right)-N^{2}\left(\frac{3}{2}+\frac{5}{2 p}\right) \\
& +N\left(3+\frac{1}{p}\right)-2
\end{aligned}
$$

respectively, where $M_{c c}(m) \quad\left(A_{c c}(m)\right)$ and $M_{s c c}(m)$ $\left(\mathrm{A}_{s c c}(m)\right)$ stand for the numbers of multiplications (additions) required for the $\mathrm{CC}$ and $\mathrm{SCC}$ matrix-vector products of size $m$, respectively.

Table 1: Comparison of row-column decomposition approach with proposed one for computing

\begin{tabular}{llcccc}
\hline Algorithms & Complexity & $3 \times 3$ & $5 \times 5$ & $7 \times 7$ & $9 \times 9$ \\
\hline Row-column & $\begin{array}{l}\text { number of } \\
\text { multiplications }\end{array}$ & 18 & 50 & 112 & 180 \\
$\begin{array}{l}\text { number of } \\
\text { additions }\end{array}$ & 24 & 130 & 420 & 612 \\
Proposed & $\begin{array}{l}\text { number of } \\
\text { multiplications } \\
\text { number of } \\
\text { additions }\end{array}$ & 8 & 30 & 64 & 80 \\
\hline
\end{tabular}

In the special case where $p=2$, the recursive equations for computing the required numbers of multiplications and additions can be simplified, respectively, as

$$
\operatorname{MUL}(N)=\left(\frac{N^{2}}{2}\right) \log _{2} N
$$

and

$$
\begin{aligned}
\operatorname{ADD}(N) & =\sum_{i=0}^{r-2}\left[\frac{N}{2^{i}}\left(\frac{3 N}{4 \times 2^{i}} \log _{2} \frac{N}{2^{i}}\right)-\frac{N}{4 \times 2^{i}}\right. \\
& \left.+\frac{N}{2 \times 2^{i}}\left(\sum_{j=0}^{r-i-1} \frac{3 \times 2^{j}}{4} \log _{2} 2^{j}+\frac{3 \times 2^{j}}{4}\right)\right] \\
& +N^{2}\left(\log _{2} N+1\right)+\sum_{i=2}^{r-1}\left(2^{i}\right)^{2}-\sum_{i=1}^{r-1} 2 p^{i}+4
\end{aligned}
$$

Table 1 provides the numbers of multiplications and additions required for computing the $p^{r} \times p^{r}$ ( $p$ is an odd prime) DCT using the proposed fast algorithm and the row-column decomposition approach, whereas other existing fast algorithms are only applicable to $p=$ 2. The 1-D DCT algorithms used in the row-column decomposition approach are based on the ones of [6], when $p=2$, and of [13], when $p$ is an odd prime, whereas the CC/SCC matrix-vector products employ the algorithms of [14]. In Table 2, we also compare the computational complexity with other existing fast algorithms when $p=2$.

As we can observe from Table 1, the proposed algorithm requires fewer numbers of multiplications (at the price of more additions), compared with those of the row-column decomposition approach. In the special case when $p=2$, we can note from Table 2 that the proposed fast algorithm calls for the same numbers of multiplications and roughly the same numbers of additions as those of $[2,4]$, which are known to be by far the most parsimonious approaches. As a whole, the proposed fast algorithm possesses the following advantageous features:

(a) The new algorithm is more general than the previous ones in that it is also applicable to the 2-D DCT of size $p^{r} \times p^{r}$, where $p$ is any prime. Although the current standards are based on the 2-D DCT of power-of-two data size, it has been observed that using a 2-D DCT of general data size can reduce the annoying blocking effect in the DCT-based image coding problems; see, for example, [15] for a discussion on lapped transforms.

(b) In contrast to the row-column decomposition approach, the new one does not require the transposi-

\begin{tabular}{|c|c|c|c|c|c|c|c|c|c|c|}
\hline \multirow{2}{*}{$\begin{array}{l}\text { Algorithms } \\
\text { Data size }\end{array}$} & \multicolumn{2}{|c|}{ Row-column } & \multicolumn{2}{|l|}{ [3] } & \multicolumn{2}{|l|}{ [2] } & \multicolumn{2}{|l|}{$[16]$} & \multicolumn{2}{|c|}{ Proposed } \\
\hline & Mult. & Add. & Mult. & Add. & Mult. & Add. & Mult. & Add. & Mult. & Add. \\
\hline $4 \times 4$ & 32 & 74 & 16 & 68 & 16 & 74 & 16 & 74 & 16 & 74 \\
\hline $8 \times 8$ & 194 & 464 & 96 & 484 & 96 & 466 & 112 & 472 & 96 & 466 \\
\hline $16 \times 16$ & 1024 & 2592 & 512 & 2531 & 512 & 2530 & 640 & 2624 & 512 & 2530 \\
\hline $32 \times 32$ & 5120 & 13376 & 2560 & 12578 & 2560 & 12738 & 3328 & 13504 & 2560 & 12738 \\
\hline $64 \times 64$ & 24576 & 65664 & 12288 & 60578 & 12288 & 61314 & 16384 & 66174 & 12288 & 61314 \\
\hline
\end{tabular}
tion manipulation. In addition, the outputs corresponding to every dominant point can be computed independently, thus allowing for efficient parallel/pipelined implementations.

(c) The 2-D to 1-D index mapping scheme employed can be readily extended to the higher-dimensional case. In contrast, the previous ones, as addressed in $[2,16]$, are specifically designed for the 2-D DCT and do not allow for any extension.

Table 2: Comparison of various fast algorithms for computing $2^{r} \times 2^{r}$ DCT 


\section{Conclusions}

In this paper, we propose a new fast algorithm for the recursive computation of a $p^{r} \times p^{r}$ DCT, where $p$ is a prime. The algorithm recursively decomposes the output components into three parts according to their indices. Such a classification of outputs can, in turn, be reformulated as a set of $\mathrm{CC}$ and $\mathrm{SCC}$ matrix-vector products of various sizes. Utilising existing fast algorithms for the computation of these $\mathrm{CC}$ and SCC matrix-vector products, we can thus obtain an algorithm with minimum multiplicative complexity. A similar fast algorithm for computing the 2-D IDCT is addressed as well.

\section{Acknowledgments}

This research was supported by the National Science Council of the Republic of China, under contract NSC87-2213-E-011-014. The authors would like to thank the reviewers, whose comments have enhanced the quality and readability of this paper.

\section{References}

1 RAO, K.R., and YIP, R.: 'Discrete cosine transform' (Academic Press, San Diego, CA, 1990)

2 CHO, N.I., and LEE, S.U.: 'Fast algorithm and implementation of 2-D discrete cosine transform', IEEE Trans. Circuits Syst., 1991, 38, (3), pp. 297-305

3 DUHAMEL, P., and GUILLEMOT, C.: 'Polynomial transform computation of the 2-D DCT'. Proceedings of IEEE international conference on Acoust., speech, signal processing, ICASSP'90, Albuquerqe, NM, Aprii 1990, pp. 1515-1518

4 DLHAMEL, P., and GUILLEMOT, C.: 'A polynomial-transform based computation of the 2-D DCT with minimum multiplicative complexity'. Proceedings of IEEE international conference on Acoust., speech, signal processing, ICASSP'96, Atlanta, GA May 1996, pp. 1347-1350

5 WU, H.R and PAOLOUI, F.J. 'A two-dimensional fast cosine transform algorithm based on Hou's approach', IEEE Trans. Signal Process., 1991, 39, (2), pp. 544-546

6 HOU, H.S.: 'A fast recursive algorithm for computing the discrete cosine transform', IEEE Trans. Acoust. Speech. Signal Process., 1987, 35, (10), pp. 1455-1461

7 KUNG, S.S, and LEE, M.H.: 'An expanded 2-D DCT algorithm based on convolution', IEEE Trans. Consum. Electron. 1993, 39, (3), pp. $159-165$

8 HU, N.-C., and LU, F.-F.: 'Fast computation of the two-dimensional generalized Hartley transforms', IEE Proc. Vis. Image Sig nal Process., 1995, 142, (1), pp. 35-39

9 GERTNER, I.: 'A new efficient algorithm to compute the twodimensional discrete Fourier transform', IEEE Trans. Acoust. Speech Signal Process, 1988 , 36, (7), pp 1036-1050

10 HU, N.-C., and LIN, K.-C.: 'Skew-circular/circular correlation decomposition of prime-factor DCT', IEE Proc. Vis. Image Signal Process., 1995, 142, (4), pp. 241-246

11 HU, N.-C., and LOUH, S.-W.: 'Two-stage decomposition of the DCT', IEE Proc. Vis. Image Signal Process., 1995, 142, (5), pp. 319--326

12 MCCLELLAN, J.H., and RADER, C.M.: 'Number theory in digital signal processing' (Prentice-Hall, Englewood Cliffs, NJ, 1979)

13 HEIDEMAN, M.T.: "Computation of an odd-length DCT from a real-valued DFT of the same length', IEEE Trans. Signal Process. 1992, 40, (1), pp. 54-61

14 NUSSBAUMER, H.H.: 'Fast Fourier transform and convolution algorithms' (Springer-Verlag, New York, 1982)

15 MALVER, H.S.: 'Signal processing with lapped transforms' (Artech House, Norwood, MA, 1992)

$16 \mathrm{CHO}$, N.I., and LEE, S.U.: 'A fast $4 \times 4$ algorithm for the recursive 2-D DCT', IEEE Trans. Signal Process., 1992, 40, (9), pp. $2166-2173$

\section{Appendix}

In this Appendix, we show the index mapping eqn. 10 For brevity, we only prove

$$
\begin{aligned}
\left(2 k_{1}+1\right) n_{1}+\left(2 k_{2}+1\right) n_{2} \equiv & (2 n+1) 2 k \quad \bmod 4 N \\
& \text { if } n_{1}+n_{2} \text { even }
\end{aligned}
$$

$$
\left(2 k_{1}+1\right) n_{1}+\left(2 k_{2}+1\right) n_{2} \equiv(2 n+1)(2 k+1) \bmod 4 N
$$

otherwise

The negative case of eqn. 10 follows straightforwardly. We begin our discussion with eqn. 34. Recall that the solution of a linear diophantine equation $a_{1} x_{1}$ $+a_{2} x_{2}=b$ can be expressed by the following parametric expression:

$$
\begin{aligned}
& x_{1}(t)=x_{1}(0)+\frac{a_{2}}{d} t \\
& x_{2}(t)=x_{2}(0)-\frac{a_{1}}{d} t
\end{aligned}
$$

where $x_{1}(0)$ and $x_{2}(0)$ are particular solutions, $d$ is the greatest common divider (GCD) of $a_{1}$ and $a_{2}$, and $t$ is an integer. As $\left(n_{0}^{1}, n_{0}^{2}\right)$ is now a dominant point with $n$ $=0$, from eqn. 5 the indices $k_{1}, k_{2}$ and $k$ need to satisfy the following expression:

$$
\left(2 k_{1}+1\right) n_{1}^{0}+\left(2 k_{2}+1\right) n_{2}^{0}=2 k \bmod 4 N
$$

A particular solution $k_{1}(0)$ and $k_{2}(0)$ to eqn. 38 can be easily determined by choosing $k_{1}(0)=0$ and then solving eqn. 38 for $k_{2}(0)$, as follows:

$$
k_{2}(0)=\frac{2 k-n_{1}^{0}-n_{2}^{0}+4 N l}{2 n_{2}^{0}}
$$

where $l$ is an integer. Employing eqns. 36 and 37, we can then obtain the general solution of eqn. 34 as

$$
\begin{gathered}
k_{1}(t)=\frac{2 n_{2}^{0}}{d} t \\
k_{2}(t)=\frac{2 k-n_{1}^{0}-n_{2}^{0}+4 N l}{2 n_{2}^{0}}-\frac{2 n_{1}^{0}}{d} t
\end{gathered}
$$

where $d=G C D\left(2 n_{1}^{0}, 2 n_{2}^{0}\right)$, and we have used the particular solution $k_{2}(0)$ of eqn. 39. Plugging the solutions $k_{1}(t)$ and $k_{2}(t)$ of eqns. 40 and 41 , respectively, in eqn. 34 and comparing both sides, we can obtain

$$
\left(2 n_{1}\right)\left(2 n_{2}^{0}\right) t-\left(2 n_{2}\right)\left(2 n_{1}^{0}\right) t=0
$$

and

$$
\frac{2 n_{2}}{2 n_{2}^{0}}\left[2 k-n_{1}^{0}-n_{2}^{0}+4 N l\right]=(2 n+1) 2 k-n_{1}-n_{2}
$$

It then follows that, for the outputs whose indices $\left(n_{1}\right.$, $\left.n_{2}\right)$ s satisfy

and

$$
n_{1} \equiv(2 n+1) n_{1}^{0} \bmod 2 N
$$

$$
n_{2} \equiv(2 n+1) n_{2}^{0} \bmod 2 N
$$

then these $\left(n_{1}, n_{2}\right)$ s share the same $\{y(k)\}$.

Similarly, when $n_{1}+n_{2}$ is odd, eqn. 35 is employed. We can also (arbitrarily) choose a particular solution as $k_{1}(0)=0$ and $k_{2}(0)=\left(2 k+1-n_{1}^{0}-n_{2}^{0}+4 N l\right) / 2 n_{2}^{0}$, where $l$ is an integer. Then, we can determine the general solution to eqn. 35 as

$$
k_{1}(t)=\frac{2 n_{2}^{0}}{d} t
$$

and

$$
k_{2}(t)=\frac{2 k-n_{1}^{0}-n_{2}^{0}+4 N l}{2 n_{2}^{0}}-\frac{2 n_{1}^{0}}{d} t
$$

Substituting eqn. 46 into eqn. 35 and equating both sides yields

$$
\left(2 n_{1}\right)\left(2 n_{2}^{0}\right) t-\left(2 n_{2}\right)\left(2 n_{1}^{0}\right) t=0
$$


and

$$
\frac{2 n_{2}}{2 n_{2}^{0}}\left[2 k-n_{1}^{0}-n_{2}^{0}\right]=(2 n+1) 2 k-n_{1}-n_{2}
$$

It then follows that, for the outputs whose indices $\left(n_{1}, n_{2}\right)$ s satisfy

$$
\begin{aligned}
& n_{1} \equiv(2 n+1) n_{1}^{0} \bmod 2 N \\
& n_{2} \equiv(2 n+1) n_{2}^{0} \bmod 2 N
\end{aligned}
$$

then these $\left(n_{1}, n_{2}\right)$ s share the same $\{y(k)\}$. Thus it completes the proof. 